\title{
Introduction to Special Issue: COVID-19 Pandemic and Its Lasting Impact on Clinical Social Work Practice and Education
}

\author{
Qingwen $\mathrm{Xu}^{1} \cdot$ Charles Figley ${ }^{2,3}$ \\ Accepted: 18 October 2021 / Published online: 25 October 2021 \\ (c) The Author(s), under exclusive licence to Springer Science+Business Media, LLC, part of Springer Nature 2021
}

When we write this introduction, it has been nearly 2 years since the first case of Covid-19 reported in Wuhan China. The Covid pandemic that ignited the world in 2020 passed 700,000 deaths in the United States. During this public health crisis, social workers have been responding to unprecedented situations and have had to adapt their practice quickly to the social, economic and political dimensions of the crisis. This Special Issue is dedicated to social workers and all practitioners who have been experiencing the pandemic and meanwhile have risked their life, their families, and their livelihood to serve others.

Since the journal announced the call for submissions for this Special Issue, we have received overwhelming responses from the social work professional and academic community; and manuscripts submitted have covered various stages of the pandemic. With all the articles, we are able to identify two primary themes: clinical practice and clinical social work education, thus present two collections for this Special Issue. Part One of the Special Issue includes contributions from practitioners and researchers, who reported and conceptualized innovative approaches to deliver clinical services to respond to the pandemic; these innovative practice models have the potential and could be the norm in the post-Covid social work field. Part Two focuses on clinical social work education; educators and students documented their collective experiences and insights in the classroom and the field that were characterized by a rapid shift from the traditional pedagogy to a new one with the incorporation of digital technology.

Qingwen Xu

qingwen.xu@nyu.edu

1 New York University Silver School of Social Work, New York, USA

2 Distinguished Chair in Disaster Mental Health, Tulane University, New Orleans, USA

3 Traumatology Institute, New Orleans, USA
The word pandemic comes from the Greek word "pan" meaning all and "demos" meaning people. The virus has stirred up the people throughout the world and measures to control the virus have profoundly changed daily routines of individuals, families and communities where they live and work. Resilience is repeatedly tested. Fear, worry, isolation, loss and uncertainty are triggering mental health conditions or worsening existing ones. People are seeking ways to cope even when their 'old' ways of coping might no longer be available.

Because of the pandemic, people have been deprived of regular contact with family members, community centers and other resources. Children have been away from school during the lockdown and families fear being cut off from accessing child and adolescent supports. Those most vulnerable live with learning disabilities, cognitive impairments, or rapid deteriorating health condition and their family members need to cope with increased care responsibilities. And there are millions of essential frontline workers-hospital health professionals, nursing home caregivers, police men and women, public transportation workers, and many other service personnel-who have coped with the ongoing pandemic without the breaks they would otherwise have received.

For clinical social work practitioners, the first and foremost challenge comes from the underpinning of these increased needs and complicated mental health conditions. These are the pandemic experiences of the people with whom social workers interact. Added to their responsibilities are their own pandemic experiences that might have contributed to their downbeat perspectives on their worklife and personal mental health. The conceptualization of shared trauma is to capture the impact of catastrophic environmental events on clinicians at multiple levels-intrapsychic, interpersonal, and community (Tosone et al., 2012). As existing vulnerabilities have been magnified in the pandemic, social workers and other practitioners from across service and geographical areas returned to these vulnerable 
groups to help without hesitation. This despite their own efforts to adapt in their own personal and family life. In this collection, both Bender et al. and Miller et al.' articles documented the shared trauma experiences. And indeed, the shared trauma scenario in the direct practice and theoretical orientation led this Special Issue.

The extraordinary breadth and depth of the articles assembled in Part One provide an example of how the practitioners recognized the importance of their work and its fit with the current pandemic. Hohman, Mc Master, and Woodruff's work describes the use of motivational interview for Covid-19 contact tracing, and demonstrates the essential role social workers played in this public health disaster. As the pandemic surely has altered the therapeutic situations, as well as the professional and personal perceptions, there has been a growing collective inspiration among scholars, educators and social work clinicians to provide trauma-related supports for clients and mental health service providers. Part One includes such newly developed clinical knowledge, strategies and skills, as well as professional reflections and insights. Among reported evidences are innovative mental health service strategies, for instance, Saidipour's "good enough therapy", and Matto and Sullivan's mindfulnessfocused creative arts to help emergency services personnel and families' coping.

Articles in Part One offer an understanding of day to day social workers' experience and their innovations during the Covid pandemic, identify good clinical practices and lessons learned, generate new working knowledge and skills, and supply preliminary guidance. More important, articles in this collection offer directions and resources for clinical practice in a hope to sufficiently support service providers to deliver excellent and targeted mental health help that match the particular population's circumstances and requirements. For instance, Bender et al. proposed strategies for "emotional connectedness" to support frontline healthcare workers; Collazo analyzed the dynamics of occupational stress and shared trauma of the pandemic to work with law enforcement couples; Craig et al. developed a telehealth version of affirmative cognitive-behavioral therapy for LGBTQ + youth; and Disney, Mowbray, and Evans discussed the use of telemental health when serving refugees.

The Covid pandemic clearly has resulted in a major transformation in the way mental health services are deliveredthe integration of technology. Telehealth has been in practice and recognized as a promising and acceptable alternative for decades (Cooper et al., 2019; Nickelson, 1998; Reay et al., 2020). While telehealth is thought to be able to deliver safe and effective care remotely and contribute to increased service accessibility and address health disparities, it never has developed and been widely adopted as with the current pace and scale; the Covid pandemic is normalizing telehealth, and technological advancements and preparation. Mishna and colleagues summarized the new trend of technology use in clinical social work practice. Also, articles in this collection discussed various approaches, formats, and arrangements of integrating technology in clinical practice. Although such integrations were not fully modeled or conceptualized, the writing offers insights to answer important questions such as: How well can technology facilitate treatments for those in need? What did clinicians do to manage chronic conditions or a long-term treatment through telecommunications? Can therapists build up a satisfied therapist-client relationship when telehealth might make therapists look/sound impersonal?

The purpose of this Special Issue is to focus on supporting direct practice efforts to address the ethical, clinical, scholarly, as well as educational needs, during and following the pandemic. When clinical social workers have to transition to telehealth during the pandemic, and likely to continue and extend the telehealth practice afterwards, social work research and training need to catch up. This is not only because in-person classroom and field learning are replaced with remote learning, but also the approaches and ways of delivering telemental health services have not been well researched, developed and then adequately included in the training curriculum. Part Two of this Special Issue is to further elaborate the clinical social work education during the Covid-19 and after.

This Special Issue is by and for the practitioners not only in the US but throughout the world who can use the wisdom and direction contained here. Social distancing and locking down would not stop our communicating with and sharing our stories with one another. The characteristics-caring, compassion, dignity, solidarity and rights that social workers have in abundance-would eventually make us strong and promote the society moving forward with the wisdom within this important and special collection.

\section{References}

Cooper, S. E., Campbell, L. F., \& Smucker Barnwell, S. (2019). Telepsychology: A primer for counseling psychologists. The Counseling Psychologist, 47(8), 1074-1114.

Nickelson, D. W. (1998). Telehealth and the evolving health care system: Strategic opportunities for professional psychology. Professional Psychology: Research and Practice, 29(6), 527.

Reay, R. E., Looi, J. C., \& Keightley, P. (2020). Telehealth mental health services during COVID-19: Summary of evidence and clinical practice. Australasian Psychiatry, 28(5), 514-516.

Tosone, C., Nuttman-Shwartz, O., \& Stephens, T. (2012). Shared trauma: When the professional is personal. Clinical Social Work Journal, 40(2), 231-239.

Publisher's Note Springer Nature remains neutral with regard to jurisdictional claims in published maps and institutional affiliations. 